\title{
PRÁTICA CLÍNICA DA EQUIPE DE ENFERMAGEM ACERCA DA TERAPIA INTRAVENOSA EM UNIDADE NEONATAL E PEDIÁTRICA
}

\author{
CLINICAL PRACTICE OF THE NURSING TEAM \\ RELATED TO INTRAVENOUS THERAPY IN A \\ NEONATAL AND PEDIATRIC UNIT
}

\section{PRÁCTICA CLÍNICA DEL EQUIPO DE ENFERMERÍA CON RESPECTO A LA TERAPIA INTRAVENOSA EN UNA UNIDAD NEONATAL Y PEDIÁTRICA}

\author{
Aline Cerqueira Santos Santana da Silva ${ }^{1}$ \\ Thaina Pinheiro das Neves e Silva ${ }^{2}$ \\ Daniel Nascimento Alves ${ }^{3}$ \\ Luiz Henrique Amarante ${ }^{4}$ \\ Fernanda Garcia Bezerra Góes ${ }^{5}$ \\ Maithê de Carvalho e Lemos Goulart ${ }^{6}$
}

Como citar este artigo: Silva ACSS, Silva TPN, Alves DN, Amarante LH, Góes FGB, Goulart MCL. Prática clínica da equipe de enfermagem acerca da terapia intravenosa em Unidade Neonatal e Pediátrica. Rev baiana enferm. 2019;33:e33828.

Objetivo: analisar a prática assistencial de uma equipe de enfermagem acerca da terapia intravenosa no setor de neonatologia e pediatria. Método: estudo de abordagem quantitativa, descritivo e exploratório em um hospital público no interior do Rio de Janeiro, Brasil. A técnica de coleta de dados foi a entrevista desenvolvida entre setembro e novembro de 2017. Resultados: amostra composta por 9 (31,1\%) enfermeiros e $20(68,9 \%)$ técnicos de enfermagem. O único dispositivo intravenoso utilizado foi o cateter venoso periférico flexível (100\%) e apenas os técnicos de enfermagem (100\%) eram responsáveis pela diluição e administração de medicamentos. Conclusão: na prática assistencial da equipe de enfermagem, o cateter venoso periférico foi o dispositivo intravenoso de escolha e o esparadrapo, a única cobertura utilizada para fixação do dispositivo, além de a terapia intravenosa ser executada majoritariamente por técnicos de enfermagem, expressando ações que ignoram a legislação e os padrões de prática assistencial segura e de qualidade.

Descritores: Infusões Intravenosas. Enfermagem Pediátrica. Cateterismo Periférico.

Objective: to analyze the professional practices of a nursing team regarding intravenous therapy in the neonatology and pediatrics sector. Method: a quantitative, descriptive and exploratory study developed in a public hospital in a

\footnotetext{
Enfermeira. Doutora em Enfermagem. Professora Assistente da Universidade Federal Fluminense. Rio das Ostras, Rio de Janeiro, Brasil. alinecer20 I4@gmail.com. http://orcid.org/0000-0002-8119-3945.

Enfermeira. Universidade Federal Fluminense. Rio das Ostras, Rio de Janeiro, Brasil. http://orcid.org/0000-0002-8972-5790.

3 Estudante de Enfermagem. Universidade Federal Fluminense. Rio das Ostras, Rio de Janeiro, Brasil. http://orcid.org/0000-0003-365I-2027.

Farmacêutico Bioquímico. Doutor em Farmacologia. Professor Associado da Universidade Federal Fluminense. Rio das Ostras, Rio de Janeiro, Brasil. http://orcid. org/0000-0003-44| I-5929.

Enfermeira. Doutora em Enfermagem. Professora Adjunta da Universidade Federal Fluminense. Rio das Ostras, Rio de Janeiro, Brasil. http://orcid.org/0000-00033894-3998.

Enfermeira. Doutora em Enfermagem. Professora Adjunta da Universidade Federal Fluminense. Rio das Ostras, Rio de Janeiro, Brasil. http://orcid.org/0000-00032764-5290.
} 
country town of the Rio de Janeiro state, Brazil. The data collection technique was an interview conducted in September and November 2017. Results: the sample was composed of 9 (31.1\%) nurses and 20 (68.9\%) nursing technicians. The only intravenous device used was the flexible peripheral venous catheter (100\%) and only nursing technicians (100\%) were responsible for the dilution and administration of medications. Conclusion: in the healthcare practices of the nursing team, the device of choice was the flexible peripheral venous catheter and the adhesive tape was the only cover used for the setting, in addition to the fact that the intravenous therapy is performed mainly by nursing technicians, ignoring legislation and standards of safety and quality care practice.

Keywords: Intravenous Infusions. Pediatric Nursing. Peripheral Catheterization.

Objetivo: analizar la práctica asistencial de un equipo de enfermería con respecto a la terapia intravenosa en el sector de neonatología y pediatría. Método: investigación cuantitativa, descriptiva y exploratoria en un hospital público en una ciudad pequeña del estado de Rio de Janeiro, Brasil. Se coleccionó a los datos por medio de una entrevista hecha desde septiembre hacia noviembre, 2017. Resultados: la amuestra incluyó 9 (31,1\%) enfermeros y 20 (68,9\%) técnicos de enfermería. El único dispositivo intravenoso utilizado fue el catéter venoso periférico flexible (100\%) y los técnicos de enfermería fueron los únicos responsables por la dilución y administración de los medicamentos. Conclusión: en la práctica asistencial del equipo de enfermería, el catéter venoso periférico fue el dispositivo intravenoso de elección, y el esparadrapo fue la única cobertura utilizada para fijar el dispositivo. La terapia intravenosa fue, mayoritariamente, hecha por técnicos de enfermería, ignorando las leyes sobre los estándares de la práctica asistencial segura y de calidad.

Descriptores: Infusiones Intravenosas. Enfermería Pediátrica. Cateterismo Periférico.

\section{Introdução}

A administração de medicamentos é um tema bastante abordado no meio científico e acadêmico, não somente devido à grande aplicabilidade como meio de recuperação do paciente na prática clínica da enfermagem, mas também devido ao amplo espectro de risco e complicações envolvidas nesse processo. Todavia, particularmente quando se trata do paciente pediátrico, a segurança e eficácia desse procedimento demanda conhecimento e atenção de toda a equipe multiprofissional, desde o estabelecimento das demandas desse paciente, prescrição e preparo, até a administração das drogas necessárias e a vigilância sobre os efeitos adversos ${ }^{(1)}$.

A Terapia Intravenosa (TI) tornou-se um recurso indispensável na prática clínica e de extrema importância na terapêutica para tratamento de doenças agudas e crônicas, além da recuperação da saúde. Tem sido utilizada para diversas finalidades, tais como em distúrbios hidroeletrolíticos, perdas sanguíneas, processos infecciosos, entre outros ${ }^{(2)}$. Atualmente, cerca de 90\% dos pacientes admitidos em unidades hospitalares têm a TI como terapêutica instituída para o restabelecimento de suas condições clínicas, o que a torna uma das formas mais comuns de intervenção no cuidado à saúde ${ }^{(3)}$.
Essa prática é caracterizada com uma das atividades mais críticas da enfermagem, especialmente no âmbito da neonatologia e da pediatria, requerendo assistência presencial do enfermeiro, profissional que está intimamente relacionado e é prioritariamente responsável pela execução dessa prática. Dessa forma, para o cuidado seguro, o conhecimento acerca do uso das tecnologias empregadas nessa terapia necessita ser expandido, não significando apenas a incorporação de equipamentos tecnológicos no cuidado, mas implicando também na necessidade de profissionais aptos a utilizá-las integralmente ${ }^{(4)}$.

No contexto da enfermagem neonatal e pediátrica, a administração de drogas injetáveis possui algumas especificidades, como cálculos específicos, dosagens mínimas e a manipulação de distintas tecnologias. Por isso, recomenda-se amplamente o uso de barreiras de segurança padronizadas, que possam impedir ou minimizar os eventos adversos advindos dessa assistência ${ }^{(5)}$.

Contudo, a TI encontra-se, muitas vezes, associada a altas incidências de complicações para o paciente submetido a esse procedimento. Por essa razão, destaca-se a importância da adoção de boas práticas que resultam de conhecimentos e habilidades necessários para o estabelecimento 
e a manutenção de uma prática segura e de qualidade frente ao preparo e à administração de medicamentos, visando prevenir e controlar essas complicações ${ }^{(6-7)}$.

Desse modo, a equipe de enfermagem desempenha um papel fundamental na prevenção de tais complicações. Sua ação visa reduzir sua incidência e possibilitar uma assistência segura e de qualidade, tendo em vista que são episódios preveníveis. Tendo em vista a importância da detecção precoce de sinais e sintomas sugestivos, é indispensável que os pacientes sejam observados diariamente ${ }^{(8)}$. Em síntese, a TI, no cenário de cuidados aos recém-nascidos e crianças, apresenta-se como um dos procedimentos que exige alto nível de complexidade técnico-científica, conhecimento e habilidade profissional por quem a realiza.

O presente estudo tem como objetivo analisar a prática assistencial de uma equipe de enfermagem acerca da terapia intravenosa no setor de neonatologia e pediatria.

\section{Método}

Trata-se de um estudo descritivo e exploratório, de abordagem quantitativa, desenvolvido na unidade de internação pediátrica e neonatal de um hospital público localizado no interior do estado do Rio de Janeiro, Brasil.

O cenário do estudo possui alta rotatividade de pacientes, recebendo não somente a população do município onde está localizado, como também de regiões vizinhas, configurando-se como porta de entrada para unidades solicitantes. A referida organização foi elencada por ser de grande porte e possuir maternidade de referência da região. Além disso, conta com berçário, emergência pediátrica e unidade de internação pediátrica, que atendem ampla variedade de diagnósticos clínicos.

A equipe de enfermagem é composta por quatro técnicos de enfermagem e um enfermeiro que se dividem entre a emergência e a unidade pediátrica, por serem setores interligados. Três técnicos ficam na emergência e um na unidade pediátrica, sob a supervisão de um enfermeiro. No berçário, ficam dois técnicos e um enfermeiro. Todos atuam sob o regime da escala de plantão de 24 horas trabalhadas por 96 horas de repouso remunerado mais 16 horas de complementação.

O número de participantes na pesquisa limitou-se ao total de profissionais existentes nos setores supracitados que atenderam aos critérios de inclusão, exclusão e disponibilidade. A amostra do estudo foi composta por 29 profissionais da equipe de enfermagem, sendo 9 enfermeiros e 20 técnicos de enfermagem. Destes 3 estavam de férias, 5 de licença médica e os demais não quiseram ou não puderam participar por indisponibilidade de tempo.

Consideraram-se como critérios de inclusão: possuidores do título de bacharelado em enfermagem e curso técnico em enfermagem; lotados nos setores de unidade de internação neonatal e pediátrica; atuantes nos referidos setores por, no mínimo, três meses (esperava-se que o participante estivesse adaptado e compreendesse o processo de trabalho no cenário em questão independentemente do tempo de formação em enfermagem). Foram excluídos: profissionais de licença de qualquer tipo e aqueles que desenvolvessem apenas atividades de cunho administrativo.

O recrutamento e a seleção dos participantes do estudo deram-se no cenário da pesquisa, em momento oportuno, em que a dinâmica do setor apresentava-se tranquila e o profissional estava livre. Na data agendada para a coleta de dados, o pesquisador apresentou-se ao pretenso participante e explicou-lhe de forma detalhada e clara em que concernia a pesquisa e os seus objetivos. Esclareceu ainda que a entrevista face a face ocorreria mediante a utilização de um roteiro estruturado, com duração média de 30 minutos e seria realizada em uma sala reservada do setor, em ambiente calmo, respeitando a privacidade desses, além de garantir-se a confidencialidade e o sigilo das informações.

A coleta de dados ocorreu no período de setembro a novembro de 2017. O instrumento foi dividido em duas partes, contendo questões sociodemográficas (idade, sexo, escolaridade, tempo de formação, tempo de atuação no setor de pediatria e berçário, dentre outros) e 
questões referentes à prática clínica da TI, tais como: dispositivo intravenoso mais utilizado, tempo de permanência do acesso venoso, medicamento mais utilizado na unidade, responsável pela diluição e administração do medicamento, entre outras. Foi realizado um teste piloto com três participantes, sendo realizadas adequações necessárias no instrumento de coleta de dados, no que tange às questões referentes à caracterização dos participantes.

Para registro dos dados coletados foi elaborada uma planilha no programa Excel, abarcando as variáveis de interesse para o estudo. Os dados foram submetidos à análise estatística descritiva, sendo calculadas a frequência, a média e o desvio padrão, utilizando-se o software Epi Info 7.1.5.0.

Em observância à Resolução no 466/2012, do Conselho Nacional de Saúde ${ }^{(9)}$, que aprova as Diretrizes e Normas Regulamentadoras de Pesquisa Envolvendo Seres Humanos, o projeto foi submetido ao Comitê de Ética em Pesquisa (CEP) da Universidade Federal Fluminense e obteve aprovação pelo Parecer no 1.570 .065 e CAEE 53503416.2.0000.5243.

\section{Resultados}

A amostra do estudo foi composta por 29 profissionais de saúde, sendo 9 (31,1\%) bacharéis em enfermagem e 20 (68,9\%) técnicos de enfermagem. A média de idade foi de 42,2 anos $( \pm 9,0)$, com maior proporção do sexo feminino $(24-82,8 \%)$. Com relação ao tempo de formação dos enfermeiros, $2(22,2 \%)$ contavam 6 a 10 anos e 7 (77,8\%), mais de 10 anos. Quanto ao tempo de atuação, houve maior prevalência na enfermagem pediátrica, superior a 10 anos, equivalendo a 13 (44,9\%), quando comparado com a enfermagem neonatal, com 5 (17,2\%).

Neste estudo, a via de administração relatada pelos $21(67,8 \%)$ respondentes como a mais usada na unidade de internação pediátrica e neonatal foi a via intravenosa. Com relação ao dispositivo intravenoso, o cateter venoso periférico flexível foi unanimemente relatado como o único utilizado. Quanto à forma de fixação deste, o esparadrapo foi citado por 18 (62,1\%) participantes como o mais utilizado. Para a variável tempo de permanência do dispositivo no paciente, um a três dias foi o tempo informado pela maior proporção de respondentes 19 (65,6\%), enquanto $9(31,0 \%)$ disseram que o mantinham enquanto durasse o acesso venoso. Do total de profissionais entrevistados, 27 (93,1\%) afirmaram que a forma de fixação do dispositivo não possibilitava a visualização do sítio de inserção, enquanto 2 (6,9\%) afirmaram visualizá-lo.

$\mathrm{Na}$ análise das variáveis relacionadas ao responsável pela diluição e administração do medicamento, verificou-se que apenas os técnicos de enfermagem eram responsáveis por essas ações. Confirmaram esse dado as declarações de $20(69,0 \%)$ profissionais de enfermagem sobre a ausência de supervisão do enfermeiro durante a diluição e administração dos medicamentos, quando realizadas pelo técnico de enfermagem.

No tocante à segurança farmacoterapêutica, quase todos os profissionais alegaram realizar ações voltadas para a segurança do paciente, desde a aquisição do medicamento até a sua administração, conforme apresentado na Tabela 1.

Tabela 1 - Distribuição das variáveis relacionadas à prática clínica da terapia intravenosa na unidade de internação neonatal e pediátrica no hospital público no interior do estado. Rio de Janeiro, RJ, Brasil - $2017(\mathrm{~N}=29)$

\begin{tabular}{|c|c|c|}
\hline Variável & $\mathbf{n}$ & $\%$ \\
\hline \multicolumn{3}{|l|}{ Via de administração } \\
\hline Intramuscular & 10 & 32,2 \\
\hline Intravenosa ou intra-arterial & 21 & 67,8 \\
\hline \multicolumn{3}{|l|}{ Dispositivo utilizado } \\
\hline Cateter venoso periférico flexível & 29 & 100,0 \\
\hline \multicolumn{3}{|l|}{ Forma de fixação do dispositivo } \\
\hline Esparadrapo & 18 & 62,1 \\
\hline Micropore & 11 & 37,9 \\
\hline
\end{tabular}


Tabela 1 - Distribuição das variáveis relacionadas à prática clínica da terapia intravenosa na unidade de internação neonatal e pediátrica no hospital público no interior do estado. Rio de Janeiro, RJ, Brasil

$-2017(\mathrm{~N}=29)$

\begin{tabular}{|c|c|c|}
\hline Variável & $\mathbf{n}$ & $\%$ \\
\hline \multicolumn{3}{|c|}{ Tempo de permanência do dispositivo no paciente (dias) } \\
\hline 1 a 3 & 19 & 65,6 \\
\hline 4 a 7 & 1 & 3,4 \\
\hline Enquanto durar o acesso & 9 & 31,0 \\
\hline \multicolumn{3}{|c|}{ Visualização do sítio de inserção pelo profissional } \\
\hline Sim & 2 & 6,9 \\
\hline Não & 27 & 93,1 \\
\hline \multicolumn{3}{|c|}{ Responsável pela diluição do medicamento } \\
\hline Técnico em enfermagem & 29 & 100,0 \\
\hline \multicolumn{3}{|c|}{ Responsável pela administração do medicamento } \\
\hline Técnico em enfermagem & 29 & 100,0 \\
\hline \multicolumn{3}{|c|}{$\begin{array}{l}\text { Supervisão pelo enfermeiro no preparo e/ou administração } \\
\text { do medicamento }\end{array}$} \\
\hline Sim & 9 & 31,0 \\
\hline Não & 20 & 69,0 \\
\hline \multicolumn{3}{|c|}{ Ações para a segurança farmacoterapêutica do paciente } \\
\hline Sim & 27 & 93,1 \\
\hline Não & 2 & 6,9 \\
\hline
\end{tabular}

Fonte: Elaboração própria.

Nos cenários deste estudo, os anti-infecciosos relatados como mais utilizados foram:
Ceftriaxona, seguida da Ampicilina e do Cefepime, conforme demonstrado no Gráfico 1.

Gráfico 1 - Anti-infecciosos utilizados na unidade de internação neonatal e pediátrica em um hospital público no interior do estado. Rio de Janeiro, RJ, Brasil, 2017 (N=29)

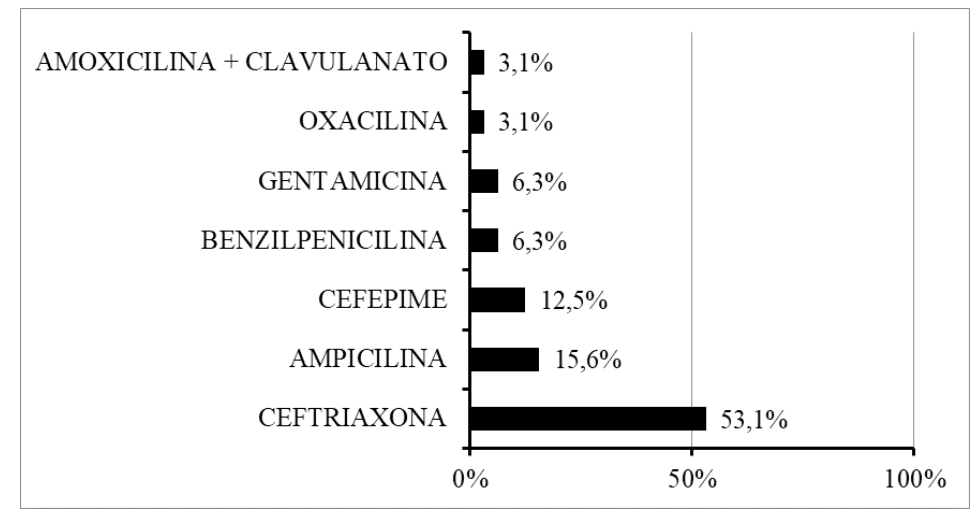

Fonte: Elaboração própria.

Com relação ao controle da dor nessas unidades, foi possível observar a adoção de medida farmacológica por meio da administração de analgésico (dipirona) como medida prevalente, quando comparadas às medidas não farmacológicas, que se apresentaram de forma menos recorrentes (Gráfico 2). 
Gráfico 2 - Adoção de medidas para controle da dor nas unidades de internação neonatal e pediátrica em um hospital público no interior do estado. Rio de Janeiro, RJ, Brasil, 2017 (N=29)

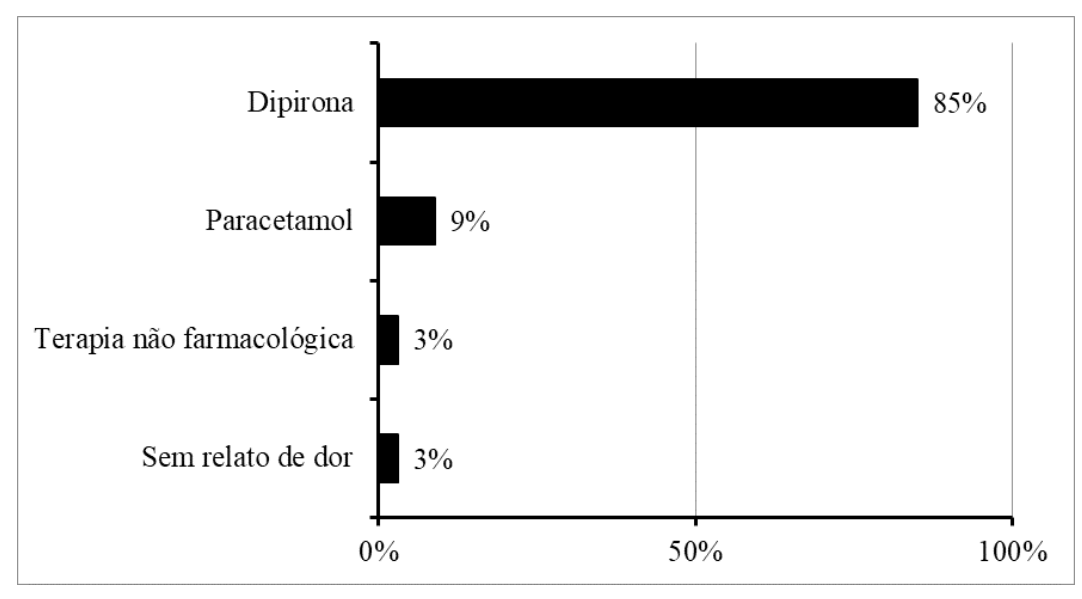

Fonte: Elaboração própria.

No que diz respeito aos medicamentos mais empregados pelos profissionais nas referidas unidades, foi utilizada a tabela de classes dos medicamentos da Anatomical Therapeutic Chemical Code (ATC) ${ }^{(10)}$, que possibilitou identificar maior frequência de anti-infecciosos para uso sistêmico, seguidos daqueles para tratamento do sistema respiratório, em relação a todas as outras classes de medicamentos (Gráfico 3).

Gráfico 3 - Frequência de uso dos medicamentos pelos profissionais de enfermagem nas unidades de internação neonatal e pediátrica em um hospital público do interior do estado. Rio de Janeiro, RJ, Brasil, $2017(\mathrm{~N}=29)$

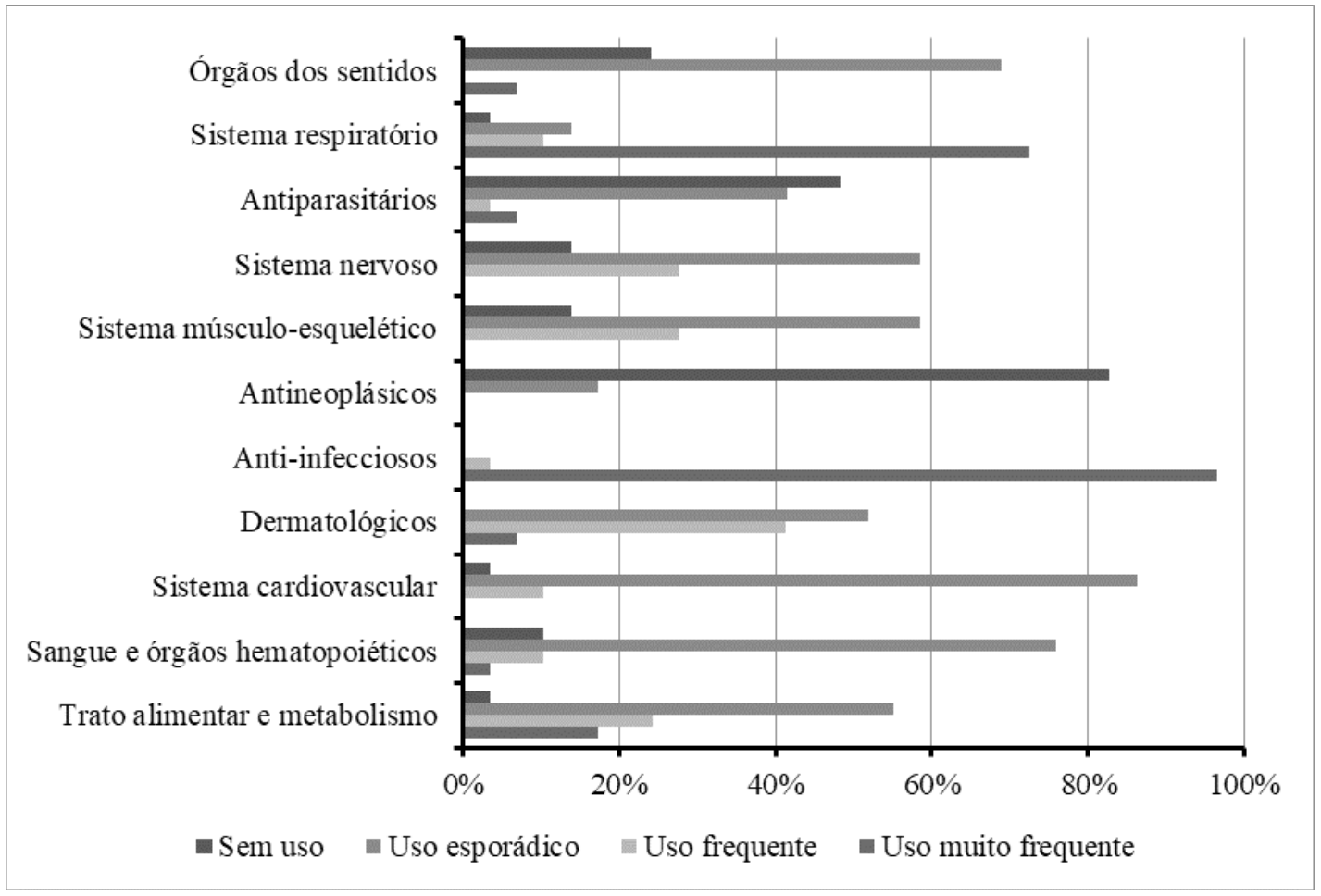

Fonte: Elaboração própria. 


\section{Discussão}

Administração de medicamentos, por si só, configura um procedimento complexo, sobretudo na medicina pediátrica e neonatal, por envolver uma série de fatores que abarcam desde a falta de padronização de drogas para esse segmento etário até a inabilidade da comunicação efetiva diante desse processo, tornando-o propenso a erros ${ }^{(11)}$. Dessa maneira, o cuidado em relação a esse procedimento não compreende apenas questões objetivas, mas alcança também dimensões subjetivas. Assim, torna-se de fundamental importância não só para a segurança e a qualidade da assistência prestada, como também para expressar respeito e zelo, conhecimento técnico-científico, ético e visão crítica sobre o exercício dessa ação de cuidado.

Por ser a terapia intravenosa um dos recursos terapêuticos mais utilizados na clínica, a via de administração parenteral destacou-se neste estudo como a mais utilizada. Este fato tem relação com a população que acessa as unidades em estudo, por se caracterizarem como locais onde normalmente a via intravenosa é a mais acessada diante de pacientes em estado clínico instável, que necessitam de intervenções imediatas e prolongadas, para a infusão de fluxos maiores e constantes, com o propósito de recuperar a sua condição clínica ${ }^{(12)}$.

Em consonância com este achado, também foi observado neste estudo que o cateter venoso periférico flexível foi o único dispositivo intravenoso utilizado na condução dessa terapia, independentemente do tipo de terapia proposto e sua duração e/ou diagnóstico médico. Este dado coaduna-se com o encontrado em estudo ${ }^{(13)}$, no qual esse tipo de dispositivo mostrou-se efetivo para suprir as necessidades dos pacientes em sua terapia. Todavia, devem ser previamente considerados alguns fatores, como duração do tratamento, tipos de infusões, características vasculares, idade do paciente, capacidade e recursos para cuidar do dispositivo.

Além desses fatores, no caso da população neonatal e pediátrica, é necessário observar algumas características que são peculiares, como a rede vascular que é menos calibrosa, contribuindo para maior dificuldade de localizar a veia a ser puncionada, além do temor com relação ao procedimento. Este fato, inclusive, impede a colaboração da criança na hora da punção venosa e dificulta a fixação do dispositivo na pele, além de incluir uma gama de fatores que podem resultar num conjunto de complicações não esperadas, como flebite e infiltrações, influenciando diretamente na escolha do dispositivo a ser utilizado ${ }^{(14)}$.

O tipo de cobertura utilizada para fixação do dispositivo intravenoso, neste estudo, foi o esparadrapo, como relatou grande proporção dos entrevistados. Ressalta-se que esse tipo de cobertura oclui o sítio de punção, impossibilitando a visualização dos profissionais de enfermagem e dificultando a avaliação e supervisão do óstio, no que se refere aos sinais flogísticos, ocultando complicações, como lesões ao redor do cateter, hematomas, oclusão, flebites, infiltração, extravasamento, além de sinais que podem indicar infecções ${ }^{(6)}$.

Cumpre destacar que, de acordo com o Centers for Disiase and Prenvention (CDC) ${ }^{(15)}$, a cobertura tem como finalidade proteger o sítio de punção e minimizar a possibilidade de infecção, permeando a interface entre a superfície do cateter e a pele, e fixar o dispositivo no local, prevenindo a movimentação com dano ao vaso. Neste sentido, toda a cobertura para cateter periférico ou profundo deve ser estéril, podendo ser semioclusiva ou membrana transparente semipermeável. O CDC ainda ressalta que a utilização de gaze e fita adesiva apenas deve ocorrer quando a previsão de acesso for inferior a 48 horas; caso contrário, não se deve utilizar gaze e esparadrapo como cobertura. Assim, é destacado que a fixação do cateter, quando realizada com esparadrapo, impossibilita a avaliação do sítio de inserção, que deve ser diária e, portanto, impede a adoção de boas práticas frente a terapia intravenosa.

Com relação ao responsável pela diluição e administração de medicamentos usados na TI, foi observado que esta ação era efetivamente desenvolvida pelo técnico de enfermagem sem 
a supervisão do enfermeiro. Contudo, quando questionados sobre a adoção de ações para a efetiva garantia da segurança farmacoterapêutica do paciente, a grande maioria dos depoentes, relatou que ocorriam.

Sendo a administração de medicamentos a forma mais comum de intervenção nos sistemas de saúde, é consenso na literatura que erros dessa ordem tornem-se frequentes, especialmente no setor de pediatria. Destaca-se que, entre todos os pacientes hospitalizados, cerca de 3\% estavam relacionados a um evento adverso grave decorrente da prática clínica com medicamentos durante o tempo de internação ${ }^{(16)}$. Diante disso, torna-se premente que os profissionais de enfermagem detenham conhecimentos e habilidades, no que tange ao preparo, às vias de administração e aos eventos adversos, além da implementação de estratégias, boas práticas e, portanto, maior responsabilidade na administração de fármacos ${ }^{(17)}$.

Desse modo, destacam-se as responsabilidades do enfermeiro frente à TI, descritas no Decreto n. 94.406, de 8 de junho de 1987, que regulamentou a Lei n. 7.498 , de 25 de junho de 1986, em seu Artigo 8, inciso II, alínea f, a qual incumbe a esse profissional participar da elaboração de medidas de prevenção e controle sistematizado de danos que possam ser causados ao paciente durante a assistência de enfermagem ${ }^{(18)}$. Nesta ação ocorre a interação complexa de fatores que envolvem o enfermeiro, o indivíduo e sua segurança.

De acordo com estudo sobre a atuação do enfermeiro na administração de medicamentos em uma instituição hospitalar ${ }^{(19)}$, a ação desse profissional relativamente à TI tem sido alvo de muitos questionamentos. Tal fato pode ser explicado devido ao conhecimento técnico científico exigido diante dessa prática e ao distanciamento do enfermeiro no tocante à supervisão de todas as etapas que abrangem a TI, em virtude de essa responsabilidade muitas vezes ser delegada ao técnico de enfermagem. Ademais, a administração de medicamentos não se caracteriza como um procedimento simples. Ao contrário, configura um processo no qual o profissional envolvido deve ter conhecimento de farmacologia, relativamente à farmacodinâmica, farmacocinética, efeitos colaterais, reações adversas, incompatibilidade, interações medicamentosas entre os fármacos administrados e, com o mesmo peso, deve-se deter conhecimento acerca do método, vias, locais, ações, diluições, além de técnicas rigorosamente assépticas ${ }^{(16)}$.

Com relação à utilização de antimicrobianos para o tratamento de morbidades, sobretudo do aparelho respiratório, foi observada, em grande proporção, a administração de Ceftriaxona, por meio de cateteres venosos periféricos flexíveis. Entretanto, pelo fato de essa medicação apresentar características vesicantes e irritantes, osmolaridade superior à osmolaridade sérica, com um Potencial de Hidrogênio ( $\mathrm{PH}$ ) baixo, inferior ao $\mathrm{PH}$ sérico $(<7)$, sua aplicação deveria se dar mediante o emprego do Cateter Central de Inserção Periférica (PICC) ${ }^{(20)}$.

Outro estudo ${ }^{(21)}$ aponta que os cateteres venosos periféricos flexíveis não devem ser utilizados para infusão contínua de produtos vesicantes, como o referido anti-infeccioso, Nutrição Parenteral (NP) com alta Taxa de Infusão de Glicose (TIG), bem como outros aditivos. O uso desse tipo de dispositivo em pacientes em estado clínico instável pode apresentar riscos iminentes, que podem levar à interrupção da terapêutica prescrita, bem como ao desenvolvimento de flebite, vaso espasmo e infecção primária da corrente sanguínea, dor, além do aumento da permanência do paciente na unidade hospitalar ${ }^{(12)}$.

Com relação ao controle da dor nos pacientes admitidos na unidade neonatal e pediátrica em estudo, foi observada maior proporção de administração de dipirona, em comparação com outros analgésicos e medidas não farmacológicas. Vale ressaltar que o controle da dor é de responsabilidade da equipe multiprofissional, em especial da equipe de enfermagem. Assim, a efetivação dessa ação deve ser preponderante durante a assistência, por meio de estratégias farmacológicas e não farmacológicas. Contudo, ao contrário do que foi evidenciado no presente estudo, evidências nacionais ${ }^{(22-24)}$ confirmam que, para o controle da dor, a implementação de 
medidas analgésicas é infrequente. Em contrapartida, as estratégias não farmacológicas privilegiam modalidades de cuidado que atuam na etapa de modulação da experiência dolorosa, subsidiadas em protocolos internacionais estabelecidos, tornando-se uma estratégia relevante no cuidado ao neonato, bem como infantil, para promover conforto e reduzir a possibilidade de erros advindos da assistência.

Ressalta-se que este estudo apresenta como limitação sua realização em uma única instituição, especialmente pelo número de pessoas investigadas, o que impede a generalização dos achados, implicando, assim, na necessidade de novos estudos sobre a temática em distintos cenários de prática.

\section{Conclusão}

A análise da prática clínica da terapia intravenosa nas unidades neonatal e pediátrica evidenciou o cateter venoso periférico flexível como de escolha, independentemente do tempo de permanência e/ou terapêutica implementada, a utilização de esparadrapo como única cobertura utilizada para o dispositivo intravenoso, além do preparo e administração de medicamentos serem realizados exclusivamente por técnicos de enfermagem, sem a supervisão do enfermeiro, expressando ações que ignoram a legislação e os padrões preconizados para a terapia intravenosa segura e de qualidade.

Considerando a complexidade e a especificidade que caracterizam a terapia intravenosa, sobretudo neonatal e pediátrica, exige-se a provisão de estruturas, processos organizacionais, bem como profissionais com alto nível de conhecimentos técnico-científicos e competência condizentes com essa prática, visando a redução de incidentes relacionados à medicação.

Esta pesquisa possui como potencialidade o retrato exposto sobre a prática clínica da terapia intravenosa num hospital público do interior do estado do Rio de Janeiro, possibilitando a análise crítica, visando a elaboração de estratégias gerenciais que possam efetivar a adoção de boas práticas, para o alcance de bons resultados em saúde, e a redução de lesões relacionadas ao preparo e à administração de medicamentos.

\section{Colaborações:}

1 - concepção, projeto, análise e interpretação dos dados: Aline Cerqueira Santos Santana da Silva, Thaina Pinheiro das Neves e Silva, Luiz Henrique e Daniel Nascimento Alves;

2 - redação do artigo e revisão crítica relevante do conteúdo intelectual: Luiz Henrique Amarantes, Daniel Nascimento Alves e Fernanda Garcia Bezerra Góes;

3 - aprovação final da versão a ser publicada: Aline Cerqueira Santos, Fernanda Garcia Bezerra e Maithê de Carvalho e Lemos Goulart.

\section{Referências}

1. Silva EMR, Souza ACC, Siqueira FOF, Silva SMO. Grau de conhecimento de profissionais de enfermagem no preparo e administração de medicamentos em pediatria. Rev em Foco. 2018 set;1(24):1-28.

2. Jacinto AKL, Avelar AFM, Wilson AMMM, Pedreira MLG. Flebite associada a cateteres intravenosos periféricos em crianças: estudo de fatores predisponentes. Esc Anna Nery. 2014 jun 18(2):220-6. DOI: http://dx.doi. org/10.5935/1414-8145.20140032

3. Lima AC. Complicações relacionadas a terapia intravenosa periférica em adultos cardiopatas internados [dissertação]. Campo Grande: Universidade Federal de Mato Grosso do Sul; 2013.

4. Moreira APA, Escudeiro CL, Christovam BP, Silvino ZR, Carvalho MF, Silva RCL. Use of technologies in intravenous therapy: contributions to a safer practice. Rev Bras Enferm. 2017 May/ Jun;70(3):595-601. DOI: http://dx.doi.org/10.1590/ 0034-7167-2016-0216

5. Julca CSM, Rocha PK, Tomazoni A, Manzo BF, Souza S, Anders JC. Utilização de barreiras de segurança no preparo de drogas vasoativas e sedativos/analgésicos em terapia intensiva pediátrica. Cogitare Enferm. 2018;23(4):e54247. DOI: http://dx.doi.org/10.5380/ce.v23i4.54247

6. Danski MTR, Oliveira GLR, Johann DA, Pedrolo E, Vayego SA. Incidência de complicações locais no cateterismo venoso periférico e fatores 
de risco associados. Acta Paul Enferm. 2015 dez;28(6):517-23. DOI: http://dx.doi.org/10.1590/ 1982-0194201500087

7. Milutinovic D, Simin D, Zec D. Fatores de risco para flebite: estudo com questionário sobre a percepção dos enfermeiros. Rev Latino-Am Enfermagem. 2015 jul-ago;23(4):677-84. DOI: http://dx.doi.org/10.1590/0104-1169.0192.2603

8. Danski MTR, Mingorance P, Johann DA, Vayego AS, Lind J. Incidência de complicações locais e fatores de risco associados ao cateter intravenoso periférico em neonatos. Rev esc enferm USP. 2016 fev;50(1):22-8. DOI: http://dx.doi.org/10.1590/ S0080-623420160000100003

9. Brasil. Conselho Nacional de Saúde. Resolução n. 466, de 12 de dezembro de 2012. Aprova diretrizes e normas regulamentadoras de pesquisas envolvendo seres humanos [Internet]. Brasília (DF); 2012 [cited 2019 Jul 17]. Available from: https://conselho.saude.gov.br/resolucoes/2012/ Reso466.pdf

10. Brasil. Agência Nacional de Vigilância Sanitária. Código ATC. Brasília; 1996 [cited 2019 Jul 18]. Available from: http://www.anvisa.gov.br/datavisa/ Substancia/ATC.htm

11. Leal KDB, Leopoldino RWD, Martins RR, Veríssimo LM. Potential intravenous drug incompatibilities in a pediatric unit. Einstein (São Paulo). 2016 Apr-Jun;14(2):185-9. DOI: 10.1590/ S1679-45082016AO3723

12. Sá Neto JAD, Siva ACSS, Vidal AR, Knupp VMAO, Barcia LLC, Barreto ACM. Conhecimento dos enfermeiros do cateter central de inserção periférica: realidade local e desafios globais. Rev enferm UERJ. 2018;26:e33181. DOI: http://dx.doi. org/10.12957/reuerj.2018.33181

13. Infusion Nurses Society. Infusion nursing standards of practice. J Infus Nurs [Internet]. 2016 [cited 2019 Jul 17];39(1S). Available from: https://source. yiboshi.com/20170417/1492425631944540325.pdf

14. Bitencourt ES, Leal CN, Boostel R, Azevedo VM, Félix JVC, Pedrolo E. Prevalência de flebite relacionada ao uso de dispositivos intravenosos periféricos em crianças. Cogitare Enferm. 2017 nov;23(1):29-38. DOI: http://dx.doi.org/10.5380/ ce.v23il.49361

15. Centers for Disease Control and Prevention. Guidelines for the prevention of intravascular catheter-related infections, 2017. Washington (DC); 2017 [cited 2019 Jul 17]. Available from: https://www.cdc.gov/infectioncontrol/guidelines/ bsi/updates.html\#anchor_1554127635

16. Belela ASC, Pedreira MLG, Peterlini MAS. Erros de medicação em pediatria. Rev bras enferm. 2011 May-Jun;64(3):563-9. DOI: http://dx.doi. org/10.1590/S0034-71672011000300022

17. Freitas RF, Pinheiro MLP, Resille DP, Campos TS. Administração de medicamentos por via parenteral: uma revisão. Rev Conexão Ciência. 2016 Jun;11(1):153-8. DOI: https://doi.org/10.24862/ cco.v11i1.342

18. Brasil. Decreto-Lei n. 94,406, de 8 de junho de 1987. Regulamenta a Lei n. 7.498, de 25 de junho de 1986, que dispõe sobre o exercício da Enfermagem, e dá outras providências [Internet]. Brasília (DF); 1987 [cited 2019 Aug 17]. Available from: http://www. cofen.gov.br/decreto-n-9440687_4173.html

19. Tomasy YT, Souza GN, Bitencourt JVOV, Parker AG, Martini JG, Manancia JR. Atuação do enfermeiro na administração de medicamentos em uma instituição hospitalar: estudo descritivo. Enferm Foco [Internet]. 2015 Jun [cited 2019 Sep 3];6(1):6-11. Available from: http://revista. cofen.gov.br/index.php/enfermagem/article/ view/569/251

20. Costa P, Paiva ED, Kimura AF, Castro TE. Fatores de risco para infecção de corrente sanguínea associada ao cateter central de inserção periférica em neonatos. Acta Paul Enferm. 2016 Abr;29(2):161-8. DOI: http://dx.doi. org/10.1590/1982-0194201600023

21. Brasil. Agência Nacional de Vigilância Sanitária. Critérios Diagnósticos de Infecção Associada à Assistência à Saúde. Brasília (DF); 2017.

22. Sposito NP, Rossato LM, Bueno M, Kimura AF, Costa T, Guedes DM. Assessment and management of pain in newborns hospitalized in a neonatal intensive care unit: a cross-sectional study. Rev Latino-Am Enfermagem. 2017 Set;12(25):e2931. DOI: $10.1590 / 1518-8345.1665 .2931$

23. Costa T, Rossato LM, Bueno M, Secco IL, Sposito NP, Harrison D, et al. Nurses' knowledge and practices regarding pain management in newborns. Rev Esc Enferm USP. 2017;51:e03210. DOI: $10.1590 / 1518-8345.1665 .2931$ 
24. Capellini VK, Daré MF, Castral TC, Christoffel MM, Leite AM, Scochi CG. Conhecimento e atitudes de profissionais de saúde sobre avaliação e manejo da dor neonatal. Rev Eletr Enf. 2014;16(2):361-9. DOI: $10.5216 /$ ree.v16i2.23611
Recebido: 10 de outubro de 2019

Aprovado: 16 de dezembro de 2019

Publicado: 17 de março de 2020

A Revista Baiana de Enfermagem utiliza a Licença Creative Commons - Atribuição-NãoComercial 4.0 Internacional. https://creativecommons.org/licenses/by-nc/4.0/ Este artigo é de acesso aberto distribuído sob os termos da Licença Creative Commons (CC BY-NC). Esta licença permite que outros remixem, adaptem e criem a partir do seu trabalho para fins não comerciais. Embora os novos trabalhos tenham de lhe atribuir o devido crédito e não possam ser usados para fins comerciais, os usuários não têm de licenciar esses trabalhos derivados sob os mesmos termos. 